\title{
FFT-Hash-II is not yet Collision-free
}

\author{
Serge Vaudenay \\ LIENS * 45 rue d’Ulm, 75230 Paris cedex 05, France
}

\begin{abstract}
In this paper, we show that the FFT-Hash function proposed by Schnorr [2] is not collision free. Finding a collision requires about $2^{24}$ computation of the basic function of FFT. This can be done in few hours on a SUN4-workstation. In fact, it is at most as strong as a one-way hash function which returns a 48 bits length value. Thus, we can invert the proposed FFT hash-furction with $2^{43}$ basic computations. Sone simple improvements of the FET hash function are also proposed to try to get rid of the weaknesses of FFT.
\end{abstract}

\section{History}

The first version of FFT-Hashing was proposed by Schnorr during the rump session of Crypto'91 [1]. This function has been shown not to be collision free at Eurocrypt'92 [3]. An improvement of the function has been proposed the same day [2] without the weaknesses discovered. However. FFT-Hashing has still some other weaknesses as it is proved in this paper.

\section{FFT-Hash-II, Notations}

The FFT-hash function is built on a basic function $\langle$.$\rangle which takes one 128-$ bits long hash block $H$ and one 128-bits long inessage block $M$, and return a 128-bits long hash block $\langle H, M\rangle$. The hash value of $n$ message blocks $M_{1}, \ldots, M_{n}$ is $<\ldots \ll I_{0}, M_{1}>, M_{2}>, \ldots, M_{n}>$ where $H_{0}$ is a constant given in hexadecimal by :

$$
H_{0}=0123456789 a b \text { cdef fedc ba98 } 76543210
$$

The basic function is defined by two one-to-one functions Rec and FT2 on the set $\left(\mathrm{GF}_{p}\right)^{1 \hat{0}}$ where $p=2^{1 \hat{6}}+1$. The concatenation $H M$ defines 1616 -bits numbers which represents 16 numbers in $\mathrm{GF}_{p}$ between 0 and $p-2$. (Rec $\left.\circ \mathrm{FT} 2 \circ \operatorname{Rec}\right)(H M)$ defines 16 numbers of $G F_{p}$. The last 8 numbers taken modulo $2^{16}$ are the result $<H, M>$.

\footnotetext{
* The Laboratoire d'Informatique de l'Ecole Normale Supérieure is a research group affiliated with the CNRS
} 
We define the following notations:

$$
\begin{aligned}
& A(M)=H_{0} M \\
& B(M)=\operatorname{Rec}(A(M)) \\
& C(M)=\operatorname{FT} 2(B(M)) \\
& D(M)=\operatorname{Rec}(C(M))
\end{aligned}
$$

So, $\left\langle H_{0}, M\right\rangle$ is the last 8 numbers of $D(M)$ taken modulo $2^{16}$. We define $X_{i}$ the $i$-th number of $X$ (from 0 to 15 ), and $X[i, j]$ the list of the $i$-th to the $j$-th number of $X$.

If $x_{i} \in \mathrm{GF}_{p}, i=0, \ldots, 15$, we define $y_{-3}=x_{13}, y_{-2}=x_{14}$, and $y_{-1}=x_{15}$. Then, following Schnorr :

$$
y_{i}=x_{i}+y_{i-1}^{*} y_{i-2}^{*}+y_{i-3}+2^{i}
$$

where $y^{*}=1$ if $y=0$ and $y^{*}=y$ otherwise. Then, we let :

$$
\operatorname{Rec}\left(x_{0} \ldots x_{15}\right)=y_{0} \ldots y_{15}
$$

If $x_{i} \in \mathrm{GF}_{\mathrm{p}}, i=0, \ldots, \bar{\tau}$, we define :

$$
y_{j}=\sum_{i=0}^{7} \omega^{i j} x_{i}
$$

where $\omega=2^{4}$. Then, we define $F T\left(x_{0}, \ldots, x_{7}\right)=y_{0}, \ldots, y_{7}$.

If $x_{i} \in \mathrm{GF}_{p}, i=0, \ldots, 15$, we define $y_{0}, y_{2}, \ldots, y_{14}=F T\left(x_{0}, x_{2}, \ldots, x_{14}\right)$ and $y_{1}, y_{3}, \ldots, y_{15}=F T\left(x_{1}, x_{3}, \ldots, x_{25}\right)$. Then, we define $\operatorname{FT2}\left(x_{0}, \ldots, x_{15}\right)=$ $y_{0}, \ldots, y_{15}$.

\section{Basic Remarks}

If we want to find a collision to the hash function. we may look for a pair $\left(x, x^{\prime}\right)$ of two 128-bits strings such that $\left\langle H_{0}, x\right\rangle=\left\langle H_{0}, x^{\prime}\right\rangle$. In fact, we will look for $x$ and $x^{\prime}$ such that $D(x)[8,15]=D\left(x^{\prime}\right)[8,15]$.

First, we notice that we have necessarily $C(x)[11,15]=C\left(x^{\prime}\right)[11,15]$. In one direction, we show that $C(x)_{i}=C\left(x^{\prime}\right)_{i}$ for $i=11 \ldots, 15$. This is due to the equation :

$$
C_{i}=D_{i}-D_{i-1}^{*} D_{i-2}^{*}-D_{i-3}-2^{i}
$$

Conversely, if we have both $C(x)[11,15]=C\left(x^{\prime}\right)[11,15]$ and $D(x)[8,10]=$ $D\left(x^{\prime}\right)[8,10]$, then we have $D(x)[8,15]=D\left(x^{\prime}\right)[8,15]$.

Moreover, we notice on the equation 1 that $B(x)[0,7]$ is a function of $x[5,7]$ only. Let us denote :

$$
B(x)[0,7]=g(x[5,7])
$$

Finally: we notice that FT2 is a linear function. 


\section{Breaking $F F T$}

\subsection{Outlines}

If we get a set of $3.2^{24}$ strings $x$ such that $C(x)[11,15]$ is a particular string $R$ chosen arbitrarily ${ }^{2}$, we will have a collision on $D(x)[8,10]$ with probability $99 \%$ thanks to the birthday paradox. We will describe an algorithm which gives some $x$ with the definitively chosen $R$ for any $x[5,7]=a b c$.

Given $a b c=x[5,7]$, we can compute $B(x)[0,7]=g(a b c)$. If we denote $y=$ $B(x)[8,15]$, the following equation is a linear equation in $y$;

$$
F T 2(g(a b c) y)[11,15]=R
$$

We can define a function $\phi_{R}$ and three vectors $U_{o}, U_{o}, L_{\theta}^{\prime \prime}$ such that

$$
\text { (2) } \Longleftrightarrow \exists \lambda, \lambda^{\prime}, \mu \quad y=\phi_{R}(a b c)+\lambda V_{e}+\lambda^{\prime} U_{e}^{\prime}+\mu L_{0}
$$

(see section 3.2 ).

Finally, the system:

$$
\left\{\begin{aligned}
x[5,7] & =a b c \\
C(x)[11,15] & =R
\end{aligned}\right.
$$

is equivalent to the system :

$$
\left\{\begin{aligned}
x[5,7] & =a b c \\
y & =\phi_{R}(a b c)+\lambda C_{t}+\lambda^{\prime} L_{e}^{\prime \prime}+\mu L_{0} \\
H_{0} x & =\operatorname{Rec}^{-1}(g(a b c) y)
\end{aligned}\right.
$$

Which is equivalent to:

$$
\left\{\begin{aligned}
y & =\phi_{R}(a b c)+\lambda U_{e}+\lambda^{\prime} U_{e}^{\prime}+\mu U_{o}^{\prime} \\
y_{13} & =a+y_{12}^{*} y_{11}^{*}+y_{10}+2^{13} \\
y_{14} & =b+y_{13}^{*} y_{12}^{*}+y_{11}+2^{14} \\
y_{15} & =c+y_{14}^{*} y_{13}^{*}+y_{12}+2^{15} \\
x[5,7] & =a b c \\
x[0,4] & =\operatorname{Rec}^{-1}(g(a b c) y)[8,12]
\end{aligned}\right.
$$

Is we substitute $y$ by the expression of the first equation in the other equations; we obtain a system of three equations of three unknown $\lambda, \lambda^{\prime}, \mu$. This system can be shown linear in $\lambda$ and $\lambda^{\prime}$ by a good choice of $U_{e}, U_{0}$ and $U_{e}^{\prime}$. Then. this system can have some solutions only if the determinant, which is a degree 2 polynomial in $\mu$ is 0 . This can gives some $\mu$. Then, the number of $\left(\lambda, \lambda^{\prime}\right)$ is almost always unique. For more details, see section 3.3 .

Finally, this gives 0 or 2 solutions $x$, with an average number of 1 for a given $a b c$. If we try $1 \leq a<p, 1 \leq b \leq 768$ and $c=2$, we have $3.2^{24} a b c$.

${ }^{2}$ For the collisions found in this paper, $R$ is the image of my phone number by $F T 2$. 


\subsection{Solving (2)}

The function $X \longmapsto F T 2(X)[11,15]$ is linear; and has a kernel of dimension 3 . If we define :

$$
\begin{aligned}
U & =(0,0,0,0,4081,256,1,61681) \\
U^{\prime} & =(0,0,0,0,65521,4352,1,0)
\end{aligned}
$$

we notice that:

$$
\begin{aligned}
F T(U) & =(482,56863,8160,57887,7682,0,0,0) \\
F T\left(U^{\prime}\right) & =(4337,61202,65503,544,61170,3855,0,0)
\end{aligned}
$$

Let us introduce the following notation :

$$
\left(x_{0}, \ldots, x_{7}\right) \times\left(y_{0}, \ldots, y_{7}\right)=\left(x_{0}, y_{0}, \ldots, x_{7}, y_{7}\right)
$$

We have $F T 2(X \times Y)=F T(X) \times F T(Y)$. Thus, we can can define:

$$
\begin{aligned}
& U_{e}=U \times 0 \\
& U_{0}^{\prime}=0 \times U \\
& U_{e}^{\prime}=U^{\prime} \times 0
\end{aligned}
$$

So, we have:

$$
\begin{aligned}
& U_{e}=(0,0,0,0,0,0,0.0,4081,0,256,0,1,0,61681,0) \\
& U_{o}=(0,0,0,0,0,0,0,0,0,4081,0,256,0,1,0,61681) \\
& U_{s}^{\prime}=(0,0,0,0,0,0,0,0,65521,0,4352,0,1,0,0,0)
\end{aligned}
$$

These vectors are a base of the kernel of $X \longmapsto F T 2(X)[11,15]$.

If $M$ denotes the matrix of $F T$, we can write it using four $4 \times 4$ blocks:

$$
M=\left(\begin{array}{ll}
W_{11} & M_{12} \\
W_{21} & W_{22}
\end{array}\right)
$$

If $x$ and $y$ are two vectors of 4 elements, we have:

$$
F T(x y)[4, \tau]=0 \Longleftrightarrow y=-M_{22}^{-1} M_{21} x
$$

Let us define :

$$
N=-M_{22}^{-1} M_{21}=\left(\begin{array}{cccc}
65281 & 4335 & 289 & 61170 \\
3823 & 8992 & 53012 & 65248 \\
8447 & 61748 & 56545 & 4335 \\
4369 & 57090 & 3823 & 256
\end{array}\right)
$$

Now, if $x$ and $y$ are two vectors of 8 elements, we have:

$$
F T 2(x y)[8,15]=0 \Longleftrightarrow y=N x^{0} \times N x^{1}
$$

Where $x=x^{0} \times x^{1}$. Let us define:

$$
\phi_{R}(a b c)=0\left(N x^{0} \times N x^{1}+y^{0}\right)
$$

where $g(a b c)=x^{0} \times x^{1}$ and $R=F T 2\left(0 y^{0}\right)[11,15]$ for an arbitrary $y^{0}$ (one's phone number for instance). Then, $\Phi_{R}(a b c)$ is a vector which begins by $g(a b c)$, and such that $F T 2\left(\phi_{R}(a b c)\right)$ ends by a constant vector $R$.

So, we have:

$$
(2) \Longleftrightarrow \exists \lambda, \lambda^{\prime}, \mu \quad y=\Phi_{R}(a b c)+\lambda U_{e}+\lambda^{\prime} U_{e}^{\prime}+\mu U_{0}
$$




\subsection{Solving (3)}

If we hope that no $y_{i}(i=11,12,13,14)$ is equal to 0 (we may ultimately test this condition, and forget the solutions $y$ which do not pass this test, but this will be very rare), the system :

$$
\left\{\begin{aligned}
y & =\phi_{R}(a b c)+\lambda U_{e}+\lambda^{\prime} U_{e}^{\prime}+\mu L_{o} \\
y_{13} & =a+y_{12}^{*} y_{11}^{*}+y_{10}+2^{13} \\
y_{14} & =b+y_{13}^{*} y_{12}^{*}+y_{11}+2^{14} \\
y_{15} & =c+y_{14}^{*} y_{13}^{*}+y_{12}+2^{15} \\
x[5,7] & =a b c \\
x[0,4] & =\operatorname{Rec}^{-1}(g(a b c) y)[8,12]
\end{aligned}\right.
$$

imply :

$$
\begin{aligned}
z_{13}+\mu & =a+\left(z_{12}+\lambda+\lambda^{\prime}\right)\left(z_{11}+256 \mu\right)+z_{10}+256 \lambda+4352 \lambda^{\prime}+2^{13} \\
z_{14}+61681 \lambda & =b+\left(z_{13}+\mu\right)\left(z_{12}+\lambda+\lambda^{\prime}\right)+\left(z_{11}+256 \mu\right)+2^{14} \\
z_{15}+61681 \mu & =c+\left(z_{14}+61681 \lambda\right)\left(z_{13}+\mu\right)+\left(z_{12}+\lambda+\lambda^{\prime}\right)+2^{15}
\end{aligned}
$$

where $z=\phi_{R}(a b c)$. If we define

$$
\begin{aligned}
& a^{\prime}=a+z_{12} z_{11}+z_{10}+2^{13}-z_{13} \\
& b^{\prime}=b+z_{13} z_{12}+z_{11}+2^{14}-z_{14} \\
& c^{\prime}=c+z_{14} z_{13}+z_{12}+2^{15}-z_{15}
\end{aligned}
$$

we have:

$$
\left(\begin{array}{ccc}
z_{11}+256 \mu+256 & z_{11}+256 \mu+4352 & a^{\prime}-\left(1-256 z_{12}\right) \mu \\
z_{13}+\mu-61681 & z_{13}+\mu & b^{\prime}+\left(256+z_{12}\right) \mu \\
61681\left(z_{13}+\mu\right)+1 & 1 & c^{\prime}-\left(61681-z_{14}\right) \mu
\end{array}\right)\left(\begin{array}{c}
\lambda \\
\lambda^{\prime} \\
1
\end{array}\right)=0
$$

This is a linear system of unknown $\lambda$ and $\lambda^{\prime}$. If this system has an equation, which determinant has to be 0 .

\subsection{Discussion}

This condition may be sufficient in most of the cases. The determinant should be a degree 3 polynomial. However, the coefficient of $\mu^{3}$ is the determinant of the following matrix :

$$
\left(\begin{array}{ccc}
256 & 256 & \left(1-256 z_{12}\right) \\
1 & 1 & -\left(256+z_{12}\right) \\
61681 & 0 & \left(61681-z_{14}\right)
\end{array}\right)
$$

which is 0 since the first line is 256 time the second.

The coefficient of $\mu^{2}$ is 0 with probability $1 / p$, this is rare. In this case, we have one solution if the equation has a degree one, and zero or $p$ solutions in the other cases. 
$\mu$ has to satisfy a degree 2 equation. If the discriminant is different from 0, it has a square root with probability $50 \%$. So, we have two different $\mu$ or no solution with probability $50 \%$, and a single solution with probability $1 / p$.

For each $\mu$, we are likely to have a uniq solution $\left(\lambda, \lambda^{\prime}\right)$. However, it is possible to have 0 or $p$ solutions, but it is rare. So, for each solution $\left(\lambda, \lambda^{\prime}, \mu\right)$, we can compute $y$ in the system (3), then $x$. Finally, we have zero or two solutions $x$ in almost all cases.

\subsection{Reduction of the Function FFT}

To sum up, we have a function $f_{R}$ such that for a given abc:

$$
f_{R}(a b c)=\{D(x)[8,10]: x[5,7]=a b c \wedge C(x)[11,15]=R\}
$$

$f_{R}(a b c)$ is a list of 0 or $2 D(x)[8,10]$ for each $x$ such that $x[5, \bar{i}]=a b c$ and $C(x)[11,15]=R$. The average of number of $x$ is 1 , so $f_{R}$ is almost a function.

The function $f_{R}$ is a kind of reduction of $F F T$ since a collision for $f_{R}$ gives a collision for FFT. We can use the birthday paradox with $f_{R}$ to get some collision. The expected complexity is $O\left(2^{24}\right)$.

We can invert $F F\left[\right.$ with $f_{R}$ to. If we are looking for $x$ such that $D(x)[8,15]=$ $z$, we can compute $R=\operatorname{Rec}^{-1}(z)[11,15]$ and look for $a b c$ such that $f_{R}(a b c)=$ $z[0,2]$. The complexity is $2^{48}$. Then. we get the $x$ required.

\section{Finding Collisions with the Birthday Paradox}

If we suppose that $f_{R}$ is like a real random function, the probability that a set $\left\{f_{R}\left(x_{i}\right)\right\}$ for $k$ different $x_{i}$ have $k$ elements is next to:

$$
e^{-\frac{k^{2}}{2 n}}
$$

where $n$ is the cardinality of the image of $f_{R}$ : when $k$ is next to $\sqrt{n}$. So. with $n=2^{48}$ and $k=3.2^{24}$. the probability is $1 \%$.

Two collisions have been found in 24 hours by a SUN4 workstation with $k=3.2^{24}$ different $x$. With the choice :

$$
R=572617 f c b 115 c 5 c 0 a 631
$$

We got :

$F F T(17 b 32755405269152218194800 a 80002)=$ FFT $(9 c 70504 e$ 834cb15c f $40494 e 202 a 70002)=$

$0851393 d 37 c 966 e 3 d 809 d 8065 e 8 c 05 b 8$

and :

$F F T(8 c c c 23 a 4086 d$ fbb9 85f4 $70 b 2029 e 0002)=$ FFT(9d53 45ae 3286 ada7 8c77 $987702 b 40002)=$ $10 e \overline{5} 49 f 5$ adfo d91b 0450 afcc fba4 2063 


\section{Conclusion}

The main weakness of FFT-Hash-II are described in section 2. First, the beginning of the computation depends on too few information of the input : $B(x)[0, \bar{T}]$ is a function of $x[5,7]$. Second, the output allows to compute too much information of the computations in FFT: $D(x)[8,15]$ allows to compute $C(x)[11,15]$. The connection between $B(x)$ and $C(x)$ is linear, this makes our attack possible.

To get rid of the first weakness, we might mix $H_{0}$ and $x$ in $A(x)$ before applying Rec. Similarly, the result of $\left\langle H_{0}, x\right\rangle$ should be the set of $D(x)_{2 i+l}$ instead of the right side.

\section{Acknowledgment}

I am happy to thank Jean-Marc Couveignes. Antolne Jolx, Ad Shamir and JACQUEs STERN from the Groupe de Recherche en Complexité et Cryptographie for any advices. I owe a lot of time to JACQUES BEIGBEDER. RONAX Keryell and all the Service des Prestations Informatiques for hardware and software advices. Finally, I should thank France Telecom to have given to me a phone number which hid so many collisions.

\section{References}

1. C. P. Schnorr. FFT-Hashing: An Efficient Cryptographic Hash Function. Presented at the rump session ot the CRYPTO:91 Conference (unpublished)

2. C. P. Schnorr. FET-Hash II, Efficient Cryptographic Hashing. Presented at the EUROCRYPT'92 Conference (unpublished)

3. T. Baritaud, H. Gilbert, M. Girault. FFT Hashing is not Collision-free. Presented at the EUROCRYPT:92 Conference (unpublished) 